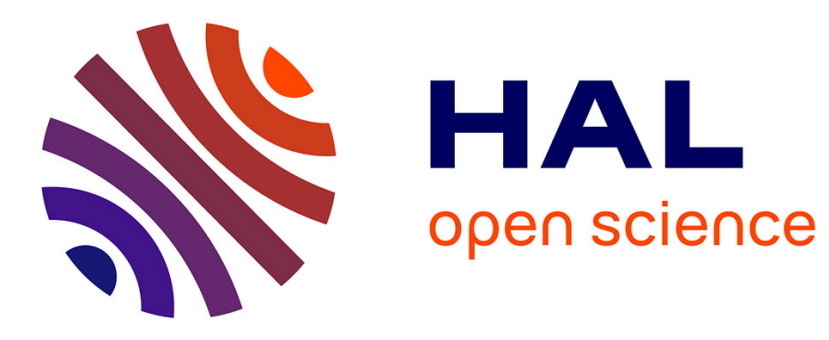

\title{
Numerical Study of Dynamo Action at Low Magnetic Prandtl Numbers
}

Yannick Ponty, Pablo Mininni, David Montgomery, Jean-François Pinton, Hélène Politano, Annick Pouquet

\section{- To cite this version:}

Yannick Ponty, Pablo Mininni, David Montgomery, Jean-François Pinton, Hélène Politano, et al.. Numerical Study of Dynamo Action at Low Magnetic Prandtl Numbers. Physical Review Letters, 2005, 94, pp.164502. ensl-00180691

\section{HAL Id: ensl-00180691}

\section{https://hal-ens-lyon.archives-ouvertes.fr/ensl-00180691}

Submitted on 19 Oct 2007

HAL is a multi-disciplinary open access archive for the deposit and dissemination of scientific research documents, whether they are published or not. The documents may come from teaching and research institutions in France or abroad, or from public or private research centers.
L'archive ouverte pluridisciplinaire HAL, est destinée au dépôt et à la diffusion de documents scientifiques de niveau recherche, publiés ou non, émanant des établissements d'enseignement et de recherche français ou étrangers, des laboratoires publics ou privés. 


\title{
Numerical Study of Dynamo Action at Low Magnetic Prandtl Numbers
}

\author{
Y. Ponty, ${ }^{1}$ P. D. Mininni, ${ }^{2}$ D. C. Montgomery, ${ }^{3}$ J.-F. Pinton, ${ }^{4}$ H. Politano, ${ }^{1}$ and A. Pouquet ${ }^{2}$ \\ ${ }^{1}$ CNRS UMR6202, Laboratoire Cassiopée, Observatoire de la Côte d'Azur, BP 4229, Nice Cedex 04, France \\ ${ }^{2}$ ASP/NCAR, P.O. Box 3000, Boulder, Colorado 80307-3000, USA \\ ${ }^{3}$ Department of Physics and Astronomy, Dartmouth College, Hanover, New Hampshire 03755, USA \\ ${ }^{4}$ CNRS UMR5672, Laboratoire de Physique, École Normale Supérieure de Lyon, 46 Allée d'Italie, 69007 Lyon, France
}

(Received 7 October 2004; published 27 April 2005)

\begin{abstract}
We present a three-pronged numerical approach to the dynamo problem at low magnetic Prandtl numbers $P_{M}$. The difficulty of resolving a large range of scales is circumvented by combining direct numerical simulations, a Lagrangian-averaged model and large-eddy simulations. The flow is generated by the Taylor-Green forcing; it combines a well defined structure at large scales and turbulent fluctuations at small scales. Our main findings are (i) dynamos are observed from $P_{M}=1$ down to $P_{M}=10^{-2}$, (ii) the critical magnetic Reynolds number increases sharply with $P_{M}^{-1}$ as turbulence sets in and then it saturates, and (iii) in the linear growth phase, unstable magnetic modes move to smaller scales as $P_{M}$ is decreased. Then the dynamo grows at large scales and modifies the turbulent velocity fluctuations.
\end{abstract}

DOI: 10.1103/PhysRevLett.94.164502

PACS numbers: 47.27.Eq, 47.65.+a, 91.25.Cw

The generation of magnetic fields in celestial bodies occurs in media for which the viscosity $\nu$ and the magnetic diffusivity $\eta$ are vastly different. For example, in the interstellar medium the magnetic Prandtl number $P_{M}=$ $\nu / \eta$ has been estimated to be as large as $10^{14}$, whereas in stars such as the Sun and for planets such as the Earth, it can be very low $\left(P_{M}<10^{-5}\right.$, the value for the Earth's iron core). Similarly in liquid breeder reactors and in laboratory experiments in liquid metals, $P_{M} \ll 1$. At the same time, the Reynolds number $R_{V}=U L / \nu$ ( $U$ is the rms velocity; $L$ is the integral scale of the flow) is very large, and the flow is highly complex and turbulent, with prevailing nonlinear effects rendering the problem difficult to address. The dynamo instability is governed by the magnetohydrodynamics (MHD) equations,

$$
\begin{gathered}
\partial_{t} \mathbf{v}+\mathbf{v} \cdot \nabla \mathbf{v}=-\nabla \mathcal{P}+\mathbf{j} \times \mathbf{B}+\nu \nabla^{2} \mathbf{v}+\mathbf{F}, \\
\partial_{t} \mathbf{B}+\mathbf{v} \cdot \nabla \mathbf{B}=\mathbf{B} \cdot \nabla \mathbf{v}+\eta \nabla^{2} \mathbf{B},
\end{gathered}
$$

together with $\nabla \cdot \mathbf{v}=0, \nabla \cdot \mathbf{B}=0$, and assuming a constant mass density. Here, $\mathbf{v}$ is the velocity field, and $\mathbf{B}$ is the magnetic field. $\mathcal{P}$ is the pressure and $\mathbf{j}=\nabla \times \mathbf{B}$ is the current density. $\mathbf{F}$ is a forcing term, responsible for the generation of the flow (buoyancy and Coriolis in planets and mechanical drive in experiments).

Several mechanisms have been studied for dynamo action, both analytically and numerically, involving, in particular, the role of helicity [1] (i.e., the correlation between velocity and its curl, the vorticity) for dynamo growth at scales larger than that of the velocity, and the role of chaotic fields for small-scale growth of magnetic excitation (for a recent review, see [2]). If the stretching and folding of magnetic field lines by velocity gradients overcome dissipation, dynamo action takes place above a critical magnetic Reynolds number $R_{M}^{c}$, with $R_{M}=P_{M} R_{V}=$ $U L / \eta$. Dynamo experiments based on constrained helical flows of liquid sodium have been successful [3]. However, these experimental setups do not allow for a complete investigation of the dynamical regime, and many groups have searched to implement unconstrained dynamos [4]. Two difficulties arise: first, turbulence now becomes fully developed with velocity fluctuations reaching up to $40 \%$ of the mean; second, it is difficult to produce flows in which the helicity is more or less uniformly distributed spatially at the small scales, so that it is difficult to assess the effect of helical turbulent inverse cascades on the dynamo. Recent direct numerical simulations (DNS) address the case of randomly forced, nonhelical flows with magnetic Prandtl numbers from 1 to 0.1 . The results obtained are not unambiguous: it is shown in [5] that dynamo action can be inhibited for $P_{M}<1 / 4$, while it is observed in [2] that the dynamo threshold increases as $P_{M}^{-1 / 2}$ down to $P_{M} \sim 0.3$. Experiments made in von Kármán geometries (either spherical or cylindrical) have reached $R_{M}$ values up to 60 [6]. Also, MHD turbulence at low $P_{M}$ has been studied in the idealized context of turbulent closures [7]. In this context, turbulent dynamos are found, and the dependences of $R_{M}^{c}$ upon three quantities are studied, namely, $P_{M}$, the relative rate of helicity injection, and the forcing scale. An increase of $\sim 20 \%$ in $R_{M}^{c}$ is observed as $P_{M}$ decreases from 1 to $\sim 3 \times 10^{-5}$. Recently, the Kazantsev-Kraichnan [8] model of $\delta$-correlated velocity fluctuations has been used to study the effect of turbulence. It has been shown that the threshold increases with the rugosity of the flow field [9].

There is therefore a strong motivation to study how the dynamo threshold varies as $P_{M}$ is progressively decreased, for a given flow. In this Letter we focus on a situation where the flow forcing is not random but generates a well defined geometry at large scales, with turbulence developing naturally at small scales as the $R_{V}$ increases. This situation complements recent investigations $[2,5,9]$ and is quite relevant for planetary and laboratory flows. 
Specifically, we consider the swirling flow resulting from the Taylor-Green (TG) forcing [10]:

$$
\mathbf{F}_{\mathrm{TG}}\left(k_{0}\right)=2 F\left[\begin{array}{c}
\sin \left(k_{0} x\right) \cos \left(k_{0} y\right) \cos \left(k_{0} z\right) \\
-\cos \left(k_{0} x\right) \sin \left(k_{0} y\right) \cos \left(k_{0} z\right) \\
0
\end{array}\right] .
$$

This force generates flow cells that have locally differential rotation and helicity, two key ingredients for dynamo action $[1,2]$. In our numerical resolutions, we have chosen not to impose on the flow field the symmetries of the Taylor-Green forcing [11] and to set $k_{0}=2$ so that dynamo action is free to develop at scales larger or smaller than the forcing scale $k_{f}=k_{0} \sqrt{3}$. The flow generated by the TG force has motivated several numerical studies in the regime $P_{M} \sim 1$ [11,12]; it also shares similarities, particularly at large Reynolds numbers, with the Maryland, Cadarache, and Wisconsin sodium experiments [4]. Its global integrated helicity is zero over the whole domain. However local fluctuations of helicity are always present in the flow-here, we mean that the probability density function of kinetic helicity is centered about zero but has wings (growing with $R_{V}$ ); but even in one TG cell the net helicity (integrated over space) is zero.

Our numerical study begins with DNS in a 3D periodic domain. The code uses a pseudospectral algorithm, an explicit second order Runge-Kutta advance in time, and a classical dealiasing rule - the last resolved wave number is $k=N / 3$ where $N$ is the number of grid points per dimension. Resolutions from $64^{3}$ to $512^{3}$ grid points are used, to cover $P_{M}$ from 1 to $1 / 5$. However, DNS are limited in the Reynolds numbers and the (lowest) $P_{M}$ they can reach. We then use a second method, the LAMHD (or $\alpha$ ) model, in which we integrate the Lagrangian-averaged MHD equations $[13,14]$. This formulation leads to a drastic reduction in the degrees of freedom at small scales by the introduction of smoothing lengths $\alpha_{V}$ and $\alpha_{M}$. The fields are written as the sum of filtered (smoothed) and fluctuating components: $\mathbf{v}=\mathbf{u}_{\mathrm{s}}+\delta \mathbf{v}, \quad \mathbf{B}=\mathbf{B}_{\mathrm{s}}+\delta \mathbf{B}$, with $\mathbf{u}_{\mathrm{s}}=$ $G_{\alpha_{V}} \otimes \mathbf{v}, \mathbf{B}_{\mathbf{s}}=G_{\alpha_{M}} \otimes \mathbf{B}$, where “ $\otimes$ " stands for convolution and $G_{\alpha}$ is the smoothing kernel at scale $\alpha, G_{\alpha}(\mathbf{r}, t)=$ $\exp [-r / \alpha] / 4 \pi \alpha^{2} r$. Inversely, the rough fields can be written in terms of their filtered counterparts as $\mathbf{v}=$ $\left(1-\alpha_{V}^{2} \nabla^{2}\right) \mathbf{u}_{\mathbf{s}}$ and $\mathbf{B}=\left(1-\alpha_{M}^{2} \nabla^{2}\right) \mathbf{B}_{\mathbf{s}}$. In the resulting equations, the velocity and magnetic field are smoothed, but not the fields' sources, i.e., the vorticity and the current density [15]. This model has been checked in the fluid case against experiments and DNS of the Navier-Stokes equations [16], as well as in MHD [14]. Finally, in order to reach still lower $P_{M}$, we implement a large-eddy simulation (LES) model. LESs are commonly used and well tested in fluid dynamics against laboratory experiments and DNS in a variety of flow configurations [17], but their extension to MHD is still in its infancy (see, however, [18]). We use a scheme as introduced in [19], aimed at integrating the primitive MHD equations with a turbulent velocity field all the way down to the magnetic diffusion with no modeling in the induction equation but with the help of a dynamical eddy viscosity [20]:

$$
\nu(k, t)=0.27\left[1+3.58\left(k / K_{c}\right)^{8}\right] \sqrt{E_{V}\left(K_{c}, t\right) / K_{c}} ;
$$

$K_{c}$ is the cutoff wave number of the velocity field, and $E_{V}(k, t)$ is the one-dimensional kinetic energy spectrum. A consistency condition for our approach is that the magnetic field fluctuations be fully resolved when $2 \pi / K_{c}$ is smaller than the magnetic diffusive scale $\ell_{\eta} \sim L / R_{M}^{3 / 4}$.

The numerical methods, parameters of the runs, and associated characteristic quantities are given in Table I. In all cases, we first perform a hydrodynamic run, lasting about 10 turnover times, to obtain a statistically steady flow. Then we add a seed magnetic field and monitor the growth of the magnetic energy $E_{M}$ for a time that depends on the run resolution; it is of the order of 1 magnetic diffusion time $\tau_{\eta}=(2 \pi)^{2} / \eta$ at $64^{3}$, but it drops down to $\tau_{\eta} / 5$ at $512^{3}$. We define the magnetic energy growth rate as $\sigma=d \log E_{M} / d t$, computed in the linear regime ( $t$ is in units of large scale turnover time). The dynamo threshold corresponds to $\sigma=0$. For each configuration (Table I), we make several MHD simulations with different $P_{M}$, varying $\eta$, and for a fixed $R_{V}$ defined by the hydrodynamic run. We

TABLE I. Run parameters: code used, linear grid resolution $N$, Reynolds number $R_{V}$, integral length scale $L$ (defined as $L=$ $\left.2 \pi \int k^{-1} E_{V}(k) d k / \int E_{V}(k) d k\right)$, critical magnetic Reynolds number $R_{M}^{c}$, inverse magnetic Prandtl number $1 / P_{M}^{c}$, wave number $k_{\max }$ with the largest magnetic energy density, characteristic wave number $k_{D}$ of magnetic field gradients (defined as the maximum of the current density spectrum), and kinetic spectral index $\rho$ in the range $\left[k_{\max }, k_{D}\right]$ (computed as a linear estimate). The values of $\rho, L$, and $U$ used in the definitions of the Reynolds and magnetic Prandtl numbers are computed as time averages during the steady state of the hydrodynamic simulation; $k_{\max }$ and $k_{D}$ are computed as time averages during the linear regime of the dynamo simulation closest to $R_{M}^{c}$.

\begin{tabular}{lcccccccc}
\hline \hline code & $N$ & $R_{V}$ & $L$ & $R_{M}^{c}$ & $1 / P_{M}^{c}$ & $k_{\max }$ & $k_{D}$ & $\rho$ \\
\hline DNS & 64 & 30.5 & 2.15 & 28.8 & 1.06 & 2 & 5 & $\ldots$ \\
DNS & 64 & 40.5 & 2.02 & 31.7 & 1.28 & 2 & 5 & $\ldots$ \\
DNS & 64 & 128 & 1.9 & 62.5 & 2.05 & 4 & 9 & $\ldots$ \\
DNS & 128 & 275 & 1.63 & 107.9 & 2.55 & 5 & 11 & -2.15 \\
DNS & 256 & 675 & 1.35 & 226.4 & 2.98 & 7 & 21 & $\sim-5 / 3$ \\
DNS & 512 & 874.3 & 1.31 & 192.6 & 4.54 & 9 & 26 & $\sim-5 / 3$ \\
LAMHD & 64 & 280 & 1.68 & 117.3 & 2.38 & 6 & 11 & $\ldots$ \\
LAMHD & 128 & 678.3 & 1.35 & 256.6 & 2.64 & 8 & 12 & $\sim-5 / 3$ \\
LAMHD & 128 & 880.6 & 1.32 & 242.1 & 3.64 & 9 & 22 & $\sim-5 / 3$ \\
LAMHD & 256 & 1301.1 & 1.3 & 249.3 & 5.22 & 9 & 31 & $\sim-5 / 3$ \\
LAMHD & 512 & 3052.3 & 1.22 & 276.4 & 11.05 & 10 & 45 & $\sim-5 / 3$ \\
LES & 128 & 2236.3 & 1.37 & 151.9 & 14.72 & 5 & 21 & $-5 / 3$ \\
LES & 256 & 5439.2 & 1.39 & 141 & 38.57 & 5 & 31 & $-5 / 3$ \\
LES & 512 & 13395 & 1.34 & 144.4 & 96.92 & 5 & 45 & $-5 / 3$ \\
\hline \hline
\end{tabular}




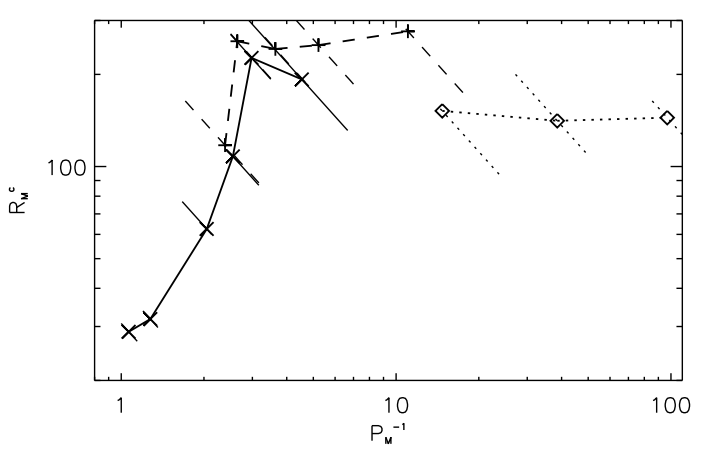

FIG. 1. $R_{M}^{c}$ for dynamo action versus inverse $P_{M}$. Symbols are $\times(\mathrm{DNS}),+(\mathrm{LAMHD})$, and $\diamond(\mathrm{LES})$. Transverse lines indicate error bars in the determination of $R_{M}^{c}$, as the distance between growing and decaying runs at a constant $R_{V}$.

bound the marginal growth between clearly decaying and growing evolutions of the magnetic energy.

At $P_{M}=1$, the dynamo self-generates at $R_{M}^{c}=30$. As $P_{M}$ is lowered, we observe in the DNS that the threshold reaches $R_{M}^{c}=70$ at $P_{M}=0.5$ and then increases steeply to $R_{M}^{c}=226$ at $P_{M}=0.3$; at lower $P_{M}$ it does not increase anymore, but drops slightly to a value of 200 at $P_{M}=0.2$ (Fig. 1 and Table I). We then continue with LAMHD simulations to reach lower $P_{M}$. To ensure the consistency of the method, we have run overlapping DNS and LAMHD simulations in the range $P_{M}=0.4-0.2$. We have reached a good matching of the growth or decay rates [for identical $\left(P_{M}, R_{M}\right)$ parameters] when using filtering scales related by $\alpha_{V} / \alpha_{M}=P_{M}^{3 / 4}$ (note that it preserves the dimensional relationship between magnetic and kinetic dissipation scales [14]). Our observation with the LAMHD computations is that the steep increase in $R_{M}^{c}$ to a value over 250 is being followed by a plateau for $P_{M}$ values down to 0.09 . We do note a small but systematic trend of the LAMHD simulations to overestimate the threshold compared to DNS. We attribute it to the increased turbulent intermittency generated by the $\alpha$ model, but further investigations are required to describe fully this effect. As turbulence becomes fully developed, LES modeling becomes justified $[17,19]$ and allows us to further our investigation; with this model we observe that the threshold for dynamo selfgeneration remains constant, of the order of $R_{M}^{c} \sim 150$, for $P_{M}$ between $10^{-1}$ and $10^{-2}$.

In regards to the generation of dynamo action in the Taylor-Green geometry we thus find (i) at all $P_{M}$ investigated a dynamo threshold exists, (ii) as $P_{M}$ drops below $0.2-0.3$, the critical $R_{M}^{c}$ levels and remains of the order of 200, and (iii) the steep initial increase in $R_{M}^{c}$ is identified with the development of an inertial range in the spectra of kinetic energy; as the kinetic energy spectrum grows progressively into a Kolmogorov $k^{-5 / 3}$ spectrum, $R_{M}^{c}$ ceases to have significant changes - cf. Table I.

We plot in Fig. 2 the magnetic energy spectra $E_{M}(k)$ during the linear growth phase, at identical instants when

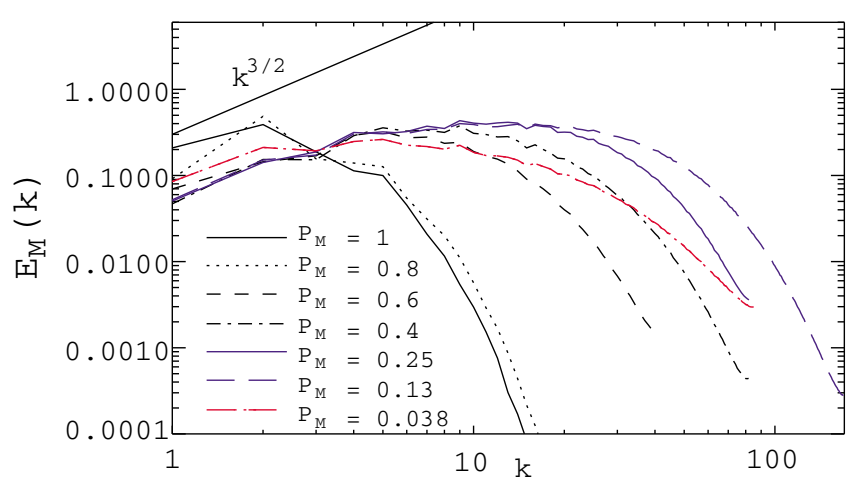

FIG. 2 (color). Magnetic spectra for $P_{M}=1$ to $P_{M}=0.4$ (DNS), $P_{M}=0.25,0.13$ (LAMHD), $P_{M}=0.038$ (LES), at a time within the linear growth of magnetic energy; $\left(P_{M}=1\right.$, $\left.R_{V}=30.5\right),\left(P_{M}=0.8, R_{V}=40.5\right),\left(P_{M}=0.6, R_{V}=275\right)$, $\left(P_{M}=0.4, R_{V}=675\right),\left(P_{M}=0.25, R_{V}=1301\right),\left(P_{M}=0.13\right.$, $\left.R_{V}=3052\right),\left(P_{M}=0.038, R_{V}=5439\right)$; magnetic Reynolds number set $10 \%$ above threshold.

normalized by the growth rate. Four features are noteworthy: first, the dynamo grows from a broad range of modes; second, the maximum of $E_{M}(k)$ moves progressively to smaller scales as $P_{M}$ decreases, a result already found numerically in [2]; third, a self-similar magnetic spectrum, $E_{M}(k) \sim k^{3 / 2}$, develops at the beginning during the linear growth phase - in a similar fashion as to Kazantsev [8] and as found in other numerical simulations of dynamo generation by turbulent fluctuations [2,5]. This feature persists when the flow has a well defined mean geometry in addition to turbulence. Last, we observe that the initial magnetic growth at small scales is always followed by a second phase where the magnetic field grows in the (large) scales of the Taylor-Green flow. Figure 3 shows renderings of the magnetic energy and compare low and high Reynolds number cases. When the dynamo is generated at low Reynolds number $\left(R_{V} \sim 30\right.$ and $\left.P_{M}=1\right)$, the magnetic field is smooth. As $P_{M}$ decreases and the dynamo grows from a turbulent field, one first observes a complex magnetic field pattern - for $t<40$, in the example shown in Fig. 3 (center). But as nonlinear effects develop (here for

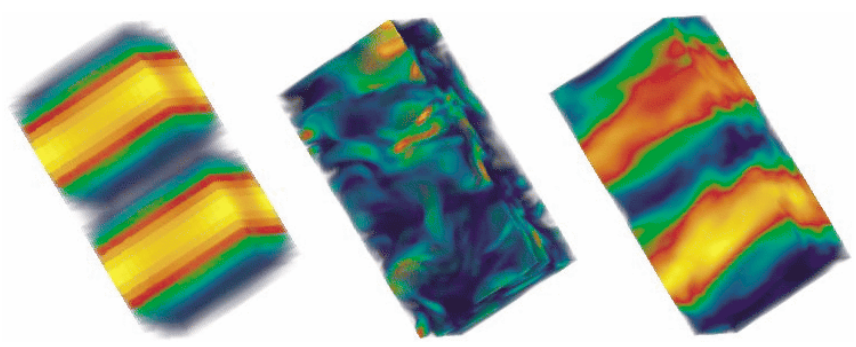

FIG. 3 (color). Spatial distributions of the magnetic energy for two Taylor-Green cells (DNS): $P_{M}=1, R_{V} \sim 30$ at $t=20$ (left), $P_{M}=0.4, R_{V}=675$ at $t=20$ (center), and $t=150$ (right). 


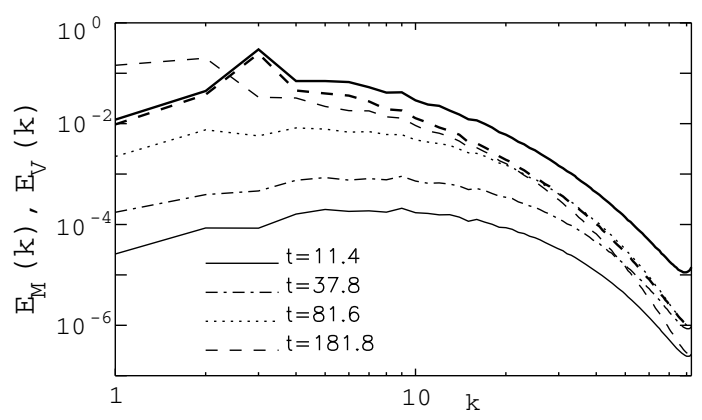

FIG. 4. Magnetic (thin line) and kinetic (thick line) spectra as a function of time at $P_{M}=0.4$ (DNS).

times $t>40)$ a large scale mode $(k=2)$ dominates the growth with a structure that is similar to the one at low $R_{V}$. The initial growth of small-scale magnetic fields and the subsequent transfer to a large scale dynamo mode is also clearly visible on the development in time of the magnetic and kinetic energies, in a high $R_{V}$ case, as shown in Fig. 4. During the linear growth, modes increase in a self-similar fashion, accounting for the complexity of the dynamo field-cf. Fig. 3 (center). At a later time, the large scale field grows and the kinetic energy spectrum $E_{V}(k)$ is progressively modified at inertial scales. The spectral slope changes from a scaling close to Kolmogorov $k^{-5 / 3}$ to a steeper decrease, compatible with the $k^{-3}$ law observed experimentally [21]. The effect is to modify the turbulent scales and to favor the dynamo mode that is allowed by the large scale flow geometry. This is consistent with the experimental observation of a $k^{-5}$ magnetic spectrum in the Karlsruhe dynamo experiment [22]. It also corroborates the claim [23] that the saturation of the turbulent dynamo starts with the backreaction of the Lorentz force on the turbulent fluctuations; the mean flow is altered at a later stage as shown in [14] where we describe the complete saturation stage.

To conclude, we have implemented a combination of DNS, LAMHD modeling, and LES. We show that for the Taylor-Green flow forcing there is a strong increase in the critical magnetic Reynolds number for dynamo action when $P_{M}$ is decreased, directly linked to the development of turbulence; it is followed by a plateau on a large range of $P_{M}$ from $\sim 0.25$ to $\sim 10^{-2}$. Thus, in a situation with both a mean flow and turbulent fluctuations, we find that the selection of the dynamo mode results from a subtle interaction between the large and small scales. Further discussions of the numerical procedure and results will be reported elsewhere [14].

We thank D. Holm for discussions about the $\alpha$ model and $\mathrm{H}$. Tufo for providing computer time at UC-Boulder, NSF ARI Grant No. CDA-9601817. NSF Grants No. ATM0327533 (Dartmouth) and No. CMG-0327888 (NCAR) are acknowledged. J.F.P., H.P., and Y.P. thank CNRS Dynamo GdR, INSU/PNST, and PCMI Programs for support. Computer time was provided by NCAR, PSC, UC,
NERSC, and IDRIS (CNRS).

[1] H. K. Moffatt, Magnetic Field Generation in Electrically Conducting Fluids (Cambridge University Press, Cambridge, 1978).

[2] N.E. L. Haugen, A. Brandenburg, and W. Dobler, Phys. Rev. E 70, 016308 (2004); A. Brandenburg and K. Subramanian, Phys. Rep. (to be published).

[3] A. Gailitis, Magnetohydrodynamics 1, 63 (1996); A. Tilgner, Phys. Rev. A 226, 75 (1997); R. Steglitz and U. Müller, Phys. Fluids 13, 561 (2001); A. Gailitis et al., Phys. Rev. Lett. 84, 4365 (2000).

[4] See the special issue on MHD dynamo experiments [Magnetohydrodynamics 38 (2002)].

[5] A. Schekochihin et al., New J. Phys. 4, 84 (2002); A. Schekochihin et al., Phys. Rev. Lett. 92, 054502 (2004).

[6] N. L. Peffley, A. B. Cawthrone, and D. P. Lathrop, Phys. Rev. E 61, 5287 (2000); M. Bourgoin et al., Phys. Fluids 14, 3046 (2002).

[7] R. H. Kraichnan and S. Nagarajan, Phys. Fluids 10, 859 (1967); J. Léorat, A. Pouquet, and U. Frisch, J. Fluid Mech. 104, 419 (1981).

[8] A. P. Kazantsev, Sov. Phys. JETP 26, 1031 (1968); R. H. Kraichnan, Phys. Fluids 11, 945 (1968).

[9] I. Rogachevskii and N. Kleeorin, Phys. Rev. E 56, 417 (1997); S. Boldyrev and F. Cattaneo, Phys. Rev. Lett. 92, 144501 (2004); D. Vincenzi, J. Stat. Phys. 106, 1073 (2002).

[10] M. Brachet, C. R. Acad. Sci. Paris Ser. IV 311, 775 (1990).

[11] N. L. Dudley and R. W. James, Proc. R. Soc. London A 425, 407 (1989); C. Nore et al., Phys. Plasmas 4, 1 (1997).

[12] L. Marié et al., Eur. Phys. J. B 33, 469 (2003); M. Bourgoin et al., Phys. Fluids 16, 2529 (2004).

[13] D. D. Holm, Physica (Amsterdam) 170D, 253 (2002).

[14] P. D. Minnini, D. C. Montgomery, and A. Pouquet, Phys. Fluids 17, 035112 (2005); Phys. Rev. E (to be published); P. D. Mininni et al., astro-ph/0412071 [Astrophys. J. (to be published)].

[15] D. C. Montgomery and A. Pouquet, Phys. Fluids 14, 3365 (2002).

[16] S. Y. Chen et al., Phys. Fluids 11, 2343 (1999); S. Y. Chen et al., Physica (Amsterdam) 133D, 66 (1999).

[17] C. Meneveau and J. Katz, Annu. Rev. Fluid Mech. 32, 1 (2000).

[18] A. Pouquet, J. Léorat, and U. Frisch, J. Fluid Mech. 77, 321 (1976); A. Yoshizawa, Phys. Fluids 30, 1089 (1987); M. Theobald, P. Fox, and S. Sofia, Phys. Plasmas 1, 3016 (1994); W.-C. Müller and D. Carati, Phys. Plasmas 9, 824 (2002); B. Knaepen and P. Moin, Phys. Fluids 16, 1255 (2004).

[19] Y. Ponty, H. Politano, and J. F. Pinton, Phys. Rev. Lett. 92, 144503 (2004).

[20] J.P. Chollet and M. Lesieur, J. Atmos. Sci. 38, 2747 (1981).

[21] A. Alemany et al., J. Mec. 18, 277 (1979).

[22] U. Müller, R. Stieglitz, and S. Horanyi, J. Fluid Mech. 498, 31 (2004).

[23] F. Pétrélis and S. Fauve, Eur. Phys. J. B 22, 273 (2001). 